\title{
ARTÍ́CULOS
}

\section{Sólidos y nanoestructuras luminiscentes}

\author{
Manuel HerRera-Zaldivar* \\ JOSÉ VALENZUELA-BENAVIDES*
}

os sólidos, al estar forLmados por átomos, los cuales a su vez contienen electrones, poseen propiedades que se explican con la ganancia y pérdida de energía de sus electrones. En este trabajo se exponen, de manera simple, algunos mecanismos de ganancia y pérdida de energía de los electrones de un semiconductor que llevan a la generación de luminiscencia. Se explica en qué consiste la técnica de catodoluminiscencia $(\mathrm{CL})$ en un microscopio electrónico de barrido (SEM) y se presenta a esta técnica como una herramienta en el estudio de las propiedades ópticas de las nanoestructuras semiconductoras.
L a energía no se crea ni se destruye, sólo se transforma. La luz es una de las formas en la que se manifiesta la energía y recibe el nombre científico de radiación electromagnética. Cuando la podemos ver con nuestros ojos la llamamos simplemente luz visible. La Figura 1 muestra la región visible del espectro de radiación electromagnética, la cual abarca desde el violeta (400 nm o $3.1 \mathrm{eV}$ ) hasta el rojo (700 nm o $1.7 \mathrm{eV}$ ), pasando por el azul (450 nm o $3.1 \mathrm{eV}$ ), verde (500 nm o $2.5 \mathrm{eV}$ ), amarillo (550 nm o 2.2 $\mathrm{eV}$ ), y naranja ( $600 \mathrm{~nm}$ o $2 \mathrm{eV}$ ). Las magnitudes que aparecen entre paréntesis son los valores de la longitud de onda y de energía de la radiación, medidos en nanómetros ( $\mathrm{nm}$ ) y en electrón-voltios $(\mathrm{eV})$, respectivamente.

Algunas veces, la luz proviene de la energía contenida en los átomos de un sólido. A esto se le conoce como el fenómeno de la luminiscencia.

Un sólido lo constituye un conjunto de átomos unidos entre sí por enlaces, que los "obligan" a mantenerse prácticamente fijos alrededor de sus

* Centro de Nanociencias y Nanotecnología, Universidad Nacional Autónoma de México, Apdo. Postal 2681, Ensenada, BC. 22800, MÉxico

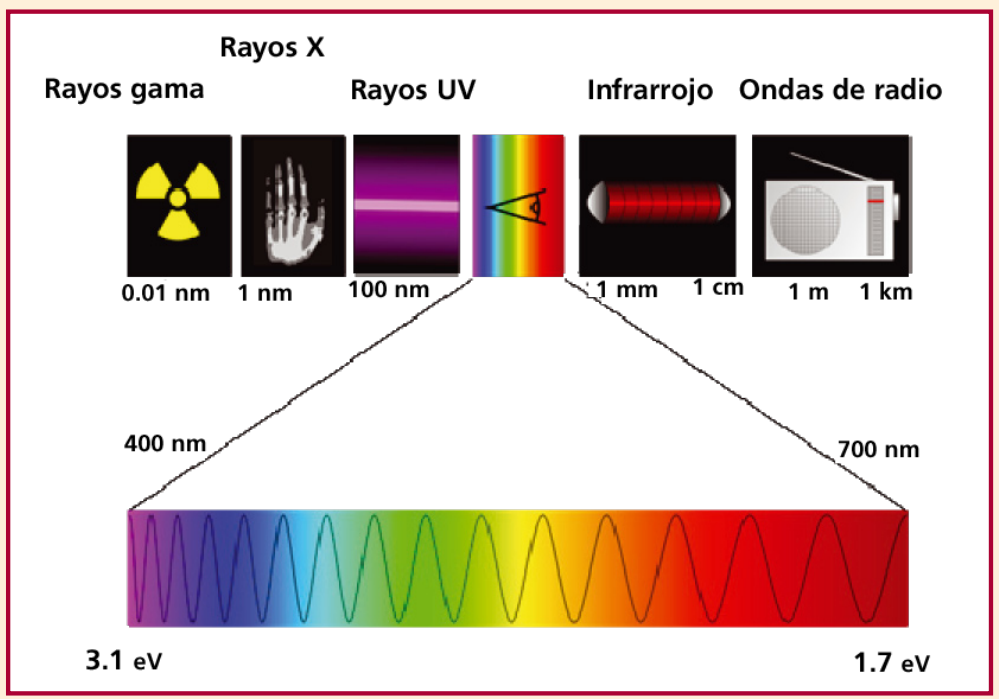

FIGURA 1. Esquema de las longitudes de onda y energías del espectro de radiación electromagnética (<http://en.wikipedia.org/wiki/Electromagnetic_radiation>) 
posiciones de equilibrio, reduciendo su movimiento a vibraciones alrededor de esas últimas. Un modelo muy usado para representar tales enlaces los considera como si fuesen resortes que unen a los átomos. Sin embargo, los enlaces químicos en realidad están formados por los electrones de los átomos del sólido. Cuando los enlaces son débiles, el sólido puede descomponerse fácilmente, o en otras palabras, es químicamente inestable.

Un átomo está formado por un núcleo, donde se concentra toda la carga positiva y casi toda su masa. Alrededor del núcleo, el cual es muy pequeño, se encuentran los electrones. Las regiones del espacio, en las cuales la probabilidad de encontrar al electrón es elevada, son conocidas como orbitales atómicos y se representan con las letras: $s, p, d, f$, etcétera.

En un sólido, los átomos poseen tanto electrones que se encuentran cerca de sus núcleos, como electrones que se encuentran lejos de ellos. A los primeros se les llama "electrones coraza" y forman una "barrera" entre los electrones alejados del núcleo y el núcleo mismo. Los electrones que quedan fuera de la coraza son llamados electrones de valencia y, al "sentir" una atracción eléctrica del núcleo atenuada por el efecto de la coraza (recordemos que el núcleo contiene protones con carga eléctrica positiva y que los electrones poseen carga eléctrica negativa), estos electrones se pueden separar ligeramente de un átomo para formar precisamente enlaces químicos con los átomos vecinos. Sin embargo, los electrones de valencia en ocasiones logran alejarse mucho de sus núcleos, como por ejemplo, por acción de un campo eléctrico aplicado al sólido para convertirse en electrones de conducción. Un sólido con capacidad de crear electrones de conducción por acción de un campo eléctrico se llama conductor. En los sólidos, en los que sus electrones de valencia no logran alejarse mucho de sus núcleos, no se formarán electrones móviles y, por tanto, el sólido será un dieléctrico. La naturaleza conductora o no-conductora de un sólido claramente depende del blindaje de la coraza de sus átomos. Mientras mayor sea el blindaje de esta coraza eléctrica mayor será la movilidad que puedan adquirir los electrones de valencia (Hall, 1978: 143).

Un sólido también puede ser conductor y no-conductor al mismo tiempo, aunque parezca paradójico. Estos sólidos se les llama semi-conductores, y la capacidad de movilidad de sus electrones de valencia depende de la energía que pueden adquirir éstos. Los electrones de valencia de un semiconductor, por un lado, "sienten" una atracción electrostática de sus núcleos atómicos, atenuada por una coraza de blindaje más bien débil; y, por otro lado, poseen una energía de movilidad propia llamada energía cinética que les "invita" a escapar de sus núcleos. La movilidad de los electrones de valencia de un semiconductor es muy sensible a los cambios de su temperatura. En los semiconductores la conducción eléctrica se reduce 


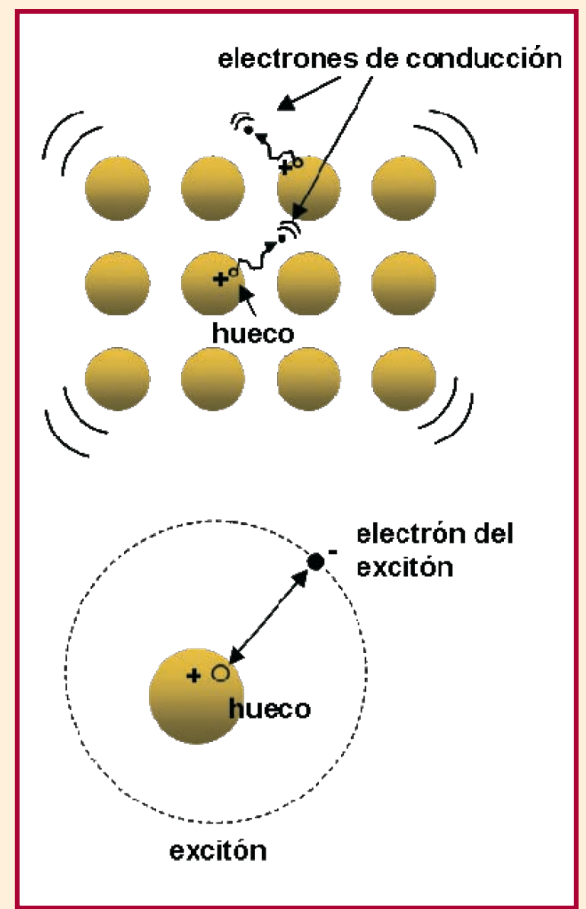

FIGURA 2. Esquema que representa la movilidad que adquieren los electrones de valencia al ganar energía y convertirse en electrones de conducción o formar un excitón. al bajar la temperatura (Shalímava, 1982: 16). Aumentar la temperatura del semiconductor equivale a incrementar su energía interna, $y$, por tanto, la movilidad de sus electrones. Con el aumento de la temperatura los electrones de valencia incrementan su energía cinética logrando incluso "vencer" la atracción de sus núcleos y escapar, convirtiéndose en electrones de conducción (Figura 2). En estas condiciones, el material se comporta como un conductor. En general, para que los electrones de valencia logren escapar de sus núcleos requieren ganar energía. Esta ganancia de energía se consigue aplicando al sólido ya sea calor (energía térmica), un campo eléctrico (energía eléctrica), luz (energía electromagnética), o haciendole incidir partículas aceleradas.

Cuando los electrones de valencia de un semiconductor ganan energía suficiente para escapar de sus núcleos, dejan en sus átomos de origen un exceso de carga positiva (Figura 2). A esta carga positiva excesiva suele llamársele hueco, pues representa una vacancia electrónica. Este hueco y el electrón libre forman un par electrón-hueco, conocido también como excitón debido a que es producido por una excitación energética. Un electrón de valencia (de carga negativa) que se convierte en un electrón libre al escapar de su orbital atómico "siente" una fuerza atractiva de su hueco (de carga positiva), que le impide migrar como un electrón de conducción, obligándolo a regresar a su órbita original, con lo cual pierde la energía cinética ganada, emitiéndola al espacio en forma de radiación electromagnética (luminiscencia). La energía de esta radiación obviamente será igual a la energía que pierde el electrón de valencia durante la recombinación con un hueco. En la mayoría de los semiconductores esta energía corresponde a luz en la región visible del espectro, y muchas veces puede observarse a simple vista debido a que en un semiconductor llegan a ocurrir miles de millones de procesos de recombinación de electrones y huecos.

En un semiconductor la energía requerida para que sus electrones de valencia se conviertan en electrones de conducción es conocida como "brecha de energía". Cada semiconductor tiene un valor característico para su brecha de energía, como se indica en la Tabla I.

La energía que los electrones de valencia pueden adquirir durante una excitación, o relajamiento, usualmente se esquematiza en lo que se conoce como estructura de bandas (Figura 3a). La banda de valencia representa los valores de la energía de los electrones de valencia. La banda de conducción 
TABLA I. Valores de la brecha de energía de algunos semiconductores

\begin{tabular}{|c|c|}
\hline Semiconductor & $\begin{array}{c}\text { Brecha de energía a } \\
\text { temperatura ambiente }\end{array}$ \\
\hline Óxido de Zinc (ZnO) & $3.2 \mathbf{e V}$ \\
\hline Arseniuro de Galio (GaAs) & $\mathbf{1 . 4} \mathbf{~ e V}$ \\
\hline Silicio (Si) & $\mathbf{1 . 1 2} \mathbf{~ e V}$ \\
\hline Germanio (Ge) & $\mathbf{0 . 6 7} \mathbf{~ V V}$ \\
\hline $\begin{array}{c}\text { Antimoniuro de Indio } \\
\text { (InSb) }\end{array}$ & $\mathbf{0 . 1 6} \mathbf{~ e V}$ \\
\hline
\end{tabular}

representa los valores de la energía que puede adquirir un electrón de valencia una vez que se convierte en un electrón libre, o de conducción. La brecha de energía se representa como una región prohibida (vacía), entre las bandas de valencia y conducción, para indicar que un electrón excitado o relajado no puede adquirir los valores de energía intermedios. La brecha de energía de los semiconductores surge por razones de la geometría del ordenamiento atómico, y por

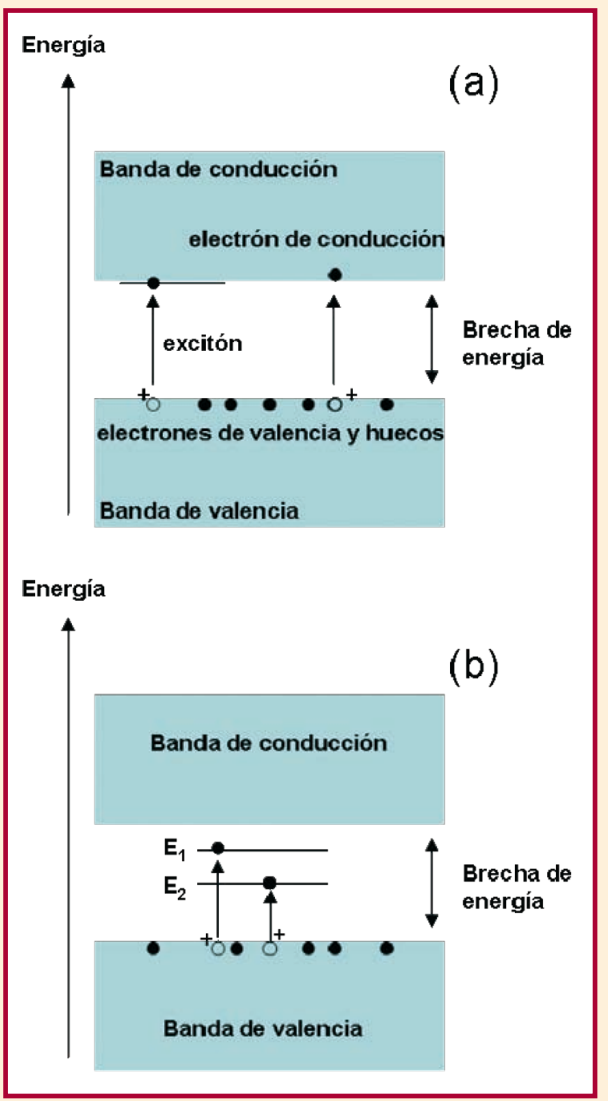

FIGURA 3. (a) Esquema de las bandas de conducción y de valencia de un semiconductor. (b) Niveles de energía $E_{1}$ y $E_{2}$ introducidos en la brecha de energía por defectos o impurezas. el tipo de orbitales de valencia que posee (Sutton, 1996: 101). Sin embargo, hay casos como el que se muestra en la Figura 3b, donde observamos dos líneas de energía en la región prohibida de un semiconductor. Esta estructura de bandas indica que sus electrones de valencia pueden adquirir los valores de energía $\mathrm{E}_{1}$ y $\mathrm{E}_{2}$ mientras ascienden a la banda de conducción, o cuando regresan a su banda de valencia. Estas líneas de energía aparecen en una brecha prohibida cuando los semiconductores poseen defectos cristalinos o impurezas atómicas.

Los defectos cristalinos y las impurezas de los semiconductores actúan como verdaderas trampas en el movimiento de los electrones. En la figura 4 se muestra, por ejemplo, a una impureza de un átomo ionizable de Sodio $^{+}\left(\mathrm{Na}^{+}\right)$, atrapando al electrón de excitón. El electrón atrapado adquiere la energía $\mathrm{E}_{1}$, que es menor que la que poseía como electrón de excitón (Figura 4a). Si el electrón de valencia atrapado por el ión $\mathrm{Na}^{+}$es liberado, entonces se recombinará con un hueco, de carga positiva, para convertirse nuevamente en un electrón de valencia con energía $E_{v}$ perdiendo una energía $\left(E_{1}-E_{v}\right)$ en forma de luminiscencia. Es claro que esta luminiscencia será de menor energía (de otro color) que la luminiscencia generada por una transición del electrón desde la banda de conducción hasta la banda de valencia.

La luminiscencia generada por electrones de valencia previamente excitados con luz de alta energía se denomina foto-luminiscencia. La producida por un campo eléctrico: electro-luminiscencia. La generada por una haz de electrones: cátodo-luminiscencia. 


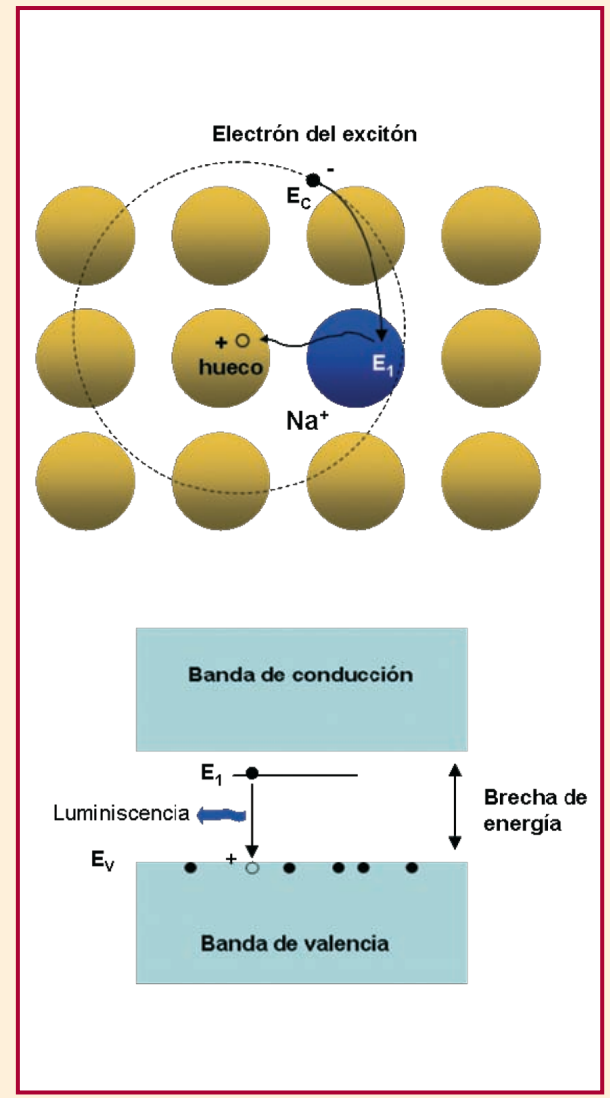

FIGURA 4. (a) Esquema de una impureza actuando como una trampa de electrones con energía $\mathrm{E}_{1 .}$ (b) Diagrama de bandas representando la generación de luminiscencia desde el nivel $\mathbf{E}_{1}$.
La cátodo-luminiscencia (CL) es una técnica que puede estar incorporada a un microscopio electrónico de barrido (SEM, abreviación del Inglés Scanning Electron Microscopy) como se muestra en la Figura 5. En un SEM, un haz de electrones incide en una pequeña región del sólido que se estudia. En esta región se hace un "barrido" de su superficie para determinar el número de electrones dispersados en una dirección dada. El detector de estos electrones (centellador en la Figura 5) está fijo dentro del microscopio y, por tanto, sólo recoge los electrones que viajan hacia ése. El número de electrones colectados durante el barrido varia de acuerdo con la topografía del sólido. Los planos cristalinos de la superficie orientados hacia el detector, contribuyen con un aumento en el número de los electrones detectados, mientras que los planos cristalinos no orientados hacia el detector hacen lo contrario. Asignando un valor de intensidad de electrones detectados en cada punto de la superficie del sólido se construye una imagen de su topografía, la cual puede llegar a alcanzar una resolución espacial de hasta 10 nanómetros (nm).

La energía del haz de electrones es típicamente de varios miles de electrón-voltios. Durante este proceso no toda la energía del haz de electrones incidente se transfiere a los electrones de valencia, ya que existen otros procesos que no se discutirán en este artículo. Sin embargo, gran cantidad de electrones de valencia sí son excitados en el semiconductor. La CL generada por el relajamiento energético de estos electrones puede colectarse dentro del microscopio (en un ambiente de alto vacío, esto es, a una presión de $10^{-5}$ Torr) mediante un arreglo óptico de lentes, o bien, mediante una fibra óptica de cuarzo (Figura 5). Esta señal óptica se debe transportar a un detector de luz, tal como un foto-diodo o un foto-multiplicador, para ser cuantificada (F en la Figura 5).

En un SEM es posible sincronizar el barrido del haz de electrones sobre una superficie con la adquisición de la señal de CL. Haciendo esto se logran asignar los valores de intensidad de luz detectada en cada punto de la superficie durante el barrido, construyendo imágenes de distribución de intensidad CL. La Figura 6(a) muestra una imagen de CL de una película semiconductora de nitruro de aluminio, indio, y galio (AlInGaN) de tan sólo $200 \mathrm{~nm}$ de espesor. Como se aprecia al comparar las imágenes de CL y topo- 
FIGURA 5. Microscopio electrónico de barrido compuesto por una columna que enfoca el haz de electrones $(A)$, cámara de análisis de la muestra (B), detector de electrones (centellador) (c), parte inferior de la columna y porta muestras, fibra óptica detector colector de la cL (D), y detector de luminiscencia tipo fotomultiplicador.

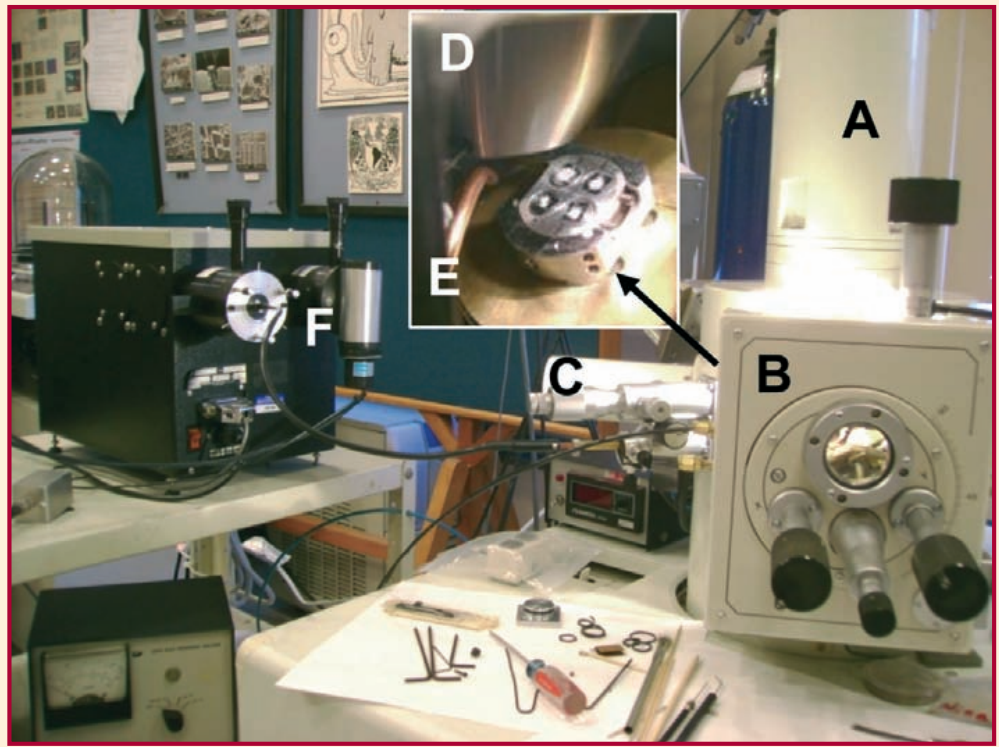

grafía (Figura 6b) las islas que componen la superficie de la película producen una intensa señal de CL. Este efecto es atribuido a un mayor contenido de Indio (In) y a una reducción de la brecha de energía del semiconductor (Herrera et al., 2004).

Las imágenes de CL nos permiten identificar las regiones de un material que producen poca o ninguna luminiscencia, lo cual es importante a la hora de localizar la formación de defectos cristalinos no deseados que inhiben la luminiscencia. La Figura 7 muestra micro-cristalitos de óxido de Galio $\left(\mathrm{Ga}_{2} \mathrm{O}_{3}\right)$ en forma de prismas, con nula o muy baja intensidad CL proveniente de sus tapas, en comparación con la registrada en sus caras.

La CL es una técnica poderosa en el estudio local de las propiedades ópticas de las nanoestructuras semiconductoras, debido a que su resolución espacial es de unos cuantos nanómetros. Mediante esta técnica es posible estudiar la luminiscencia a lo largo de un nano-cristalito individual, algo sumamente importante en el campo de la nanotecnología, la cual se propone fabricar dispositivos electrónicos a partir de uno sólo de estos nanocristalitos. Las propiedades de un sólo nano-cristal determinarán el buen funcionamiento de todo un dispositivo electrónico.

La Figura 8 es una imagen cátodo-luminiscente de nano-rodillos de óxido de zinc ( $\mathrm{ZnO}$ ) (de diámetro nanométrico) la cual muestra claramente la variación de la intensidad luminiscente en las regiones nanométricas indicadas por flechas. 

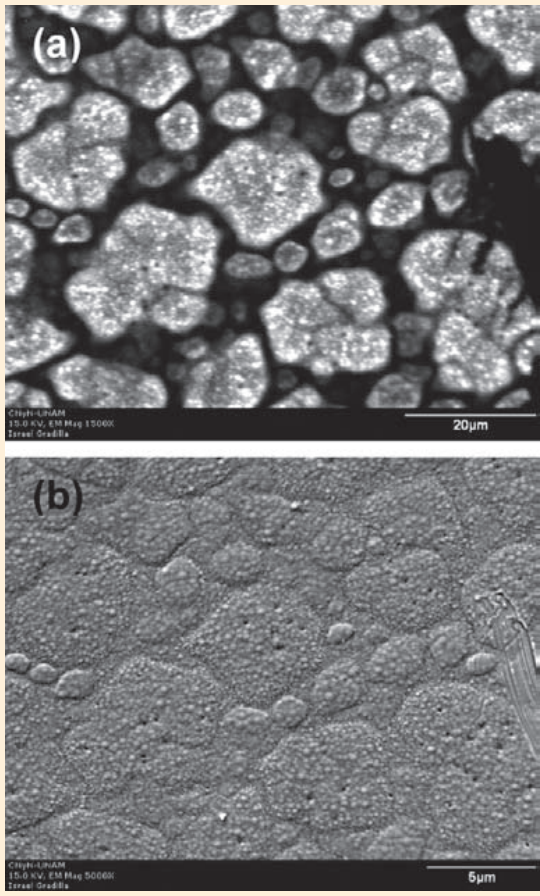

FIGURA 6. (a) Imagen de CL y (b) topografía de una película semiconductora de AllnGaN.
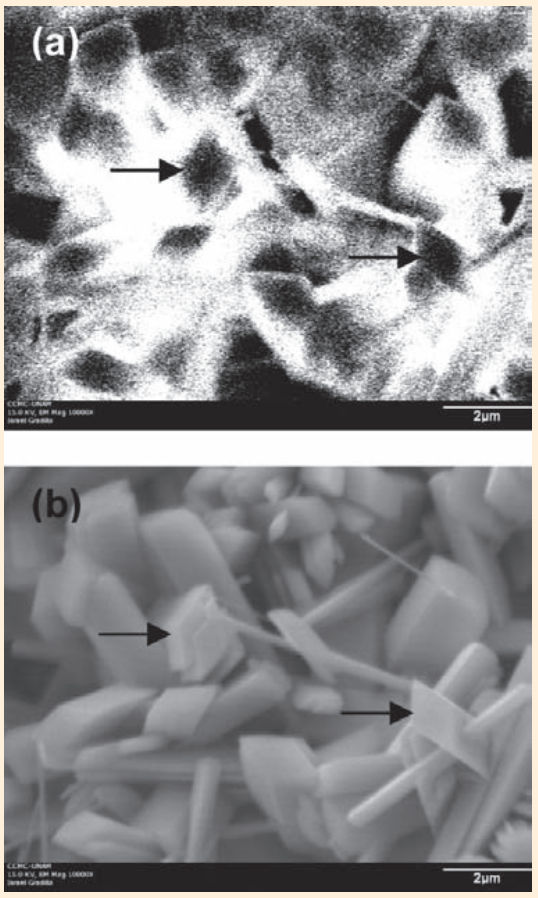

FIGURA 7. (a) Imagen de cL y (b) de topografía cristalitos de óxido de galio con forma prismática.

La resolución espacial de la CL que puede alcanzarse con un SEM está relacionada con la resolución máxima en la totografía que ofrece el instrumento. La resolución espacial de un SEM es determinada por la sensibilidad del instrumento para detectar variaciones en la intensidad de los electrones dispersados por el material en estudio, los cuales se usan para construir una imagen de topografía. Son electrones que han perdido energía durante la interacción con el sólido y son conocidos como electrones secundarios. Aunque la región de generación de los electrones secundarios tiene una profundidad de varias micras desde la superficie del sólido en donde incide el haz de electrones, solamente aquéllos con una profundidad de unos cuantos nanómetros logran escapar del sólido para ser detectados y formar la imagen de topografía. Precisamente, son los electrones secundarios los que excitan a los electrones de valencia del sólido y producen la señal de CL. Aún más, debido a que la luminiscencia es producida por la recombinación de los electrones de conducción y los huecos creados, la densidad de huecos en el sólido determinará cuánta luminiscencia es posible generar. Como 
FIGURA 8. Imágenes de cL mostrando una resolución espacial de varios nanométros en cristales de óxido de zinc (ZnO) (González et al., 2008).
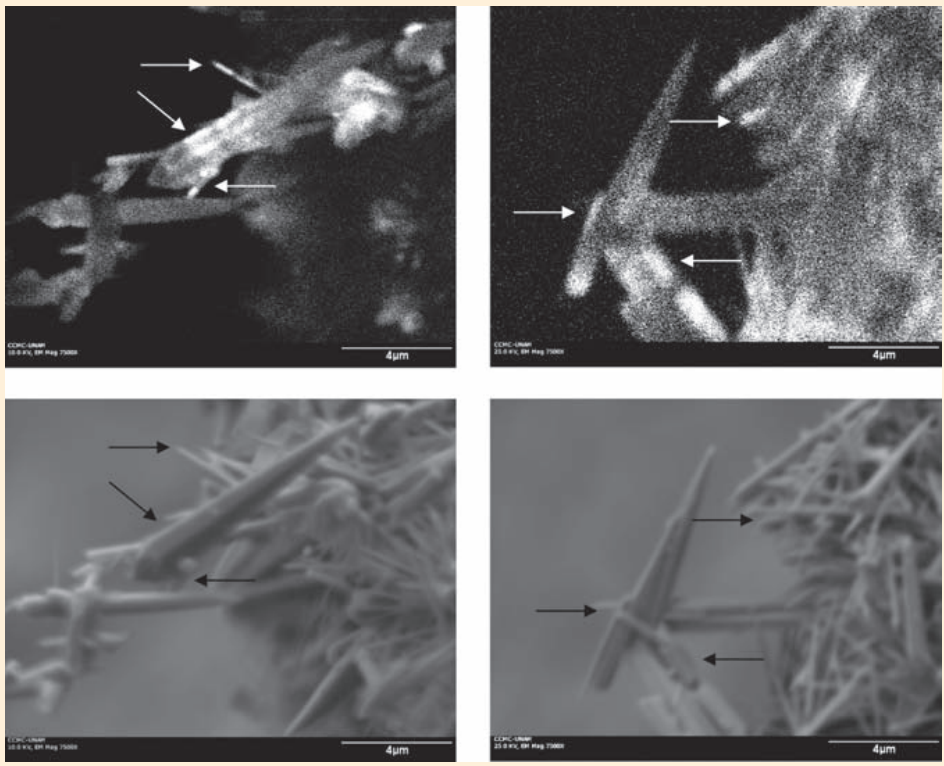

se puede uno imaginar, al poseer cargas positivas, los huecos tienen pocas posibilidades de existir en una región en donde incide un haz de electrones con miles de millones de cargas negativas. Efectivamente, se calcula que la densidad de los huecos en un semiconductor con electrones en exceso decae exponencialmente en la dirección radial del punto de incidencia del haz de electrones. Aunque esta región depende en general de la energía con que inciden los electrones del haz y de su densidad de corriente eléctrica, se calcula que es una región de tamaño inferior a la región de generación de los electrones secundarios (Yacobi, 1990), lo cual posibilita a la CL como una técnica adecuada para el estudio de las propiedades ópticas de las nanoestructuras semiconductoras.

\section{Bibliografía}

González, A., Herrera, M., Valenzuela, J., Pal, U., Escobedo, A., (2008), enviado al Journal of Applied Physics.

Hall, H. E., (1978). Física del Estado Sólido, Limusa, México.

Herrera, M., Cremades, A., Piqueras, J., Stutzmann, M., y Ambacher, $O$ (2004). Journal of Applied Physics. Volumen 95, \#10, 5305.

Shalímova, K. V. (1982). Física de los Semiconductores, Mir, URSS.

Sutton, A. P (1996). Electronic Structure of Materials, Oxford, Inglaterra.

Yacobi, B. G. y D. B. Holt (1990). Cathodoluminiscence Microscopy of Inorganic Solids. Plenum Press, New York, EUA. 